\title{
Removal of Organics in Landfill Leachate by Microwave-enhanced Fenton Process
}

\author{
Zhaolian Zhu ${ }^{1, \text { a }}$, Aixin $\mathrm{Wu}^{1}$, Hui Chen ${ }^{1}$, Bing $\mathrm{Ji}^{1}$, Hailing Wang ${ }^{1}$ \\ ${ }^{1}$ College of Environmental Science \& Engineering, Nanjing Tech University, Nanjing, 211816, China \\ aemail: zInju@163.com
}

Keywords: Landfill leachate; Organics; Fenton oxidation; Microwave

\begin{abstract}
Microwave has been used to enhance the removal of refractory organic contaminants from wastewater, but there are few studies about its use in landfill leachate. Mechanism and superiority of microwave irradiation are not completely clear in Fenton process. Here, removals of COD, $\mathrm{UV}_{254}$, chroma and fluorescence excitation-emission matrix spectroscopy (EEMs) in leachate by microwave enhanced Fenton process were investigated. The results indicated that microwave-enhanced Fenton process had much better degradation efficiency and was less affected by initial $\mathrm{pH}$ than single Fenton process due to promoting the generation rate of hydroxyl radicals and reactivity between hydroxyl radicals and organic pollutants by microwave irradiation.
\end{abstract}

\section{Introduction}

Landfill leachate is formed by the percolation of water through wastes, where water interacts with waste in an anaerobic environment, concentrating inorganic macro-components, heavy metals, dissolved organic matter (DOM), xenobiotic organic compounds and microorganisms organic matter [1][2]. Due to the complicated solid waste deposited in landfills, leachate contains a wide variety of organic compounds, where humic-type constituents consist an important group, as well as ammonia-nitrogen, heavy metals, chlorinated organic and inorganic salts. There are lots of persistent organic pollutants (POPs), endocrine disrupting chemicals (EDCs), pharmaceuticals and personal care products (PPCPs) in leachates, which may seriously pollute the groundwater aquifer and surface water, so landfill leachate must be treated to meet the local standard for discharge into natural waters.

Numerous technologies such as biological methods including aerobic biodegradation and anaerobic biodegradation, and physicochemical processes including coagulation-flocculation, chemical precipitation, membrane processes, chemical oxidation and adsorption, have been used for pretreatment, deep treatment or full treatment of leachate [3]. Among potential physicochemical technologies, the Fenton process is an attractive alternative because of its effective degradation, low toxicity, ease of operation, and the absence of mass transfer limitation due to its homogeneous nature [4]. But Fenton's process requires low $\mathrm{pH}$ and relative long reaction time, which influences the promotion and use in leachate treatment. Microwave has been widely used to enhance and accelerate the heating process and catalytic degradation activity due to its special heating mechanism. Compared with traditional heating techniques, microwave heating has advantages of rapid and selective heating, ease of control, and enhanced chemical reactivity. Recent years, many researchers have utilized microwave to enhance the removal of refractory organic contaminants from wastewater, and these investigations suggests that microwave is in favor of promoting the degradation efficiency of traditional treatment methods [5][6].

In this study, microwave enhanced Fenton process and traditional Fenton process are used to treat landfill leachate under laboratory conditions. The influences of reaction time and $\mathrm{pH}$ on COD, $\mathrm{UV}_{254}$, chroma removal and EEMs characteristics of leachate are investigated. The benefits of microwave on the oxidation efficiency of Fenton process are evaluated, and the mechanism is elucidated. 


\section{Experiment}

Landfill leachate was collected from Nanjing Tianjingwa Waste Landfill. The leachate was prefiltered througha $0.45 \mu \mathrm{m}$ glass fiber filter to remove large particles and debris, and maintain uniformity of tested samples. The leachate characteristics are as following: COD is $12363 \mathrm{mg} / \mathrm{L}, \mathrm{pH}$ value is 7.82, chroma is 1024 times, and $U_{254}$ is $18.7 \mathrm{~cm}^{-1}$. The following reagents were obtained from Aladdin Industrial Corpration at the specified purities and were used as received without further purification: $\mathrm{FeSO}_{4} \cdot 7 \mathrm{H}_{2} \mathrm{O}, \mathrm{H}_{2} \mathrm{O}_{2}$ (30\% solution), $\mathrm{H}_{2} \mathrm{SO}_{4}(98 \%)$, and $\mathrm{NaOH}$.

Traditional Fenton process was conducted by using a series of $250 \mathrm{~mL}$ flasks which containing Fenton reagents and $100 \mathrm{~mL}$ leachate. The dosage of $\mathrm{FeSO}_{4} \cdot 7 \mathrm{H}_{2} \mathrm{O}$ and $\mathrm{H}_{2} \mathrm{O}_{2}$ were $0.2 \mathrm{mg} / \mathrm{L}$ and $0.3 \mathrm{ml} / \mathrm{L}$, respectively. The mixed solutions were stirred by a magnetic stirrer at $240 \mathrm{rpm}$ for given contacting times. The microwave-enhanced Fenton process was carried out in an ordinary family microwave oven equipped with a condenser tube. The dosage and ratio were the same with Fenton process. The microwave power is $800 \mathrm{~W}$ and frequency is $2450 \mathrm{MHz}$. Afterwards, samples were withdrawn and $\mathrm{NaOH}$ solution (5M) was added to quench hydrogen peroxide in order to avoid interferences on the analytical methods and to promote iron precipitation before the subsequent analysis.

The values of COD and chroma were measured according to standard methods [7]. The $\mathrm{pH}$ value and $\mathrm{UV}_{254}$ of samples were measured with a $\mathrm{pH}$ meter and a $721 \mathrm{UV}-\mathrm{Vis}$ spectrophotometer, respectively. The EEMs of leachate were measured by fluorescence spectrometer (Perkin Elmer Luminescence Spectrometer LS50B, USA).

\section{Results and discussion}

Fig. 1 shows the traditional Fenton process and microwave enhanced Fenton process for leachate treatment. When the reaction time is $10 \mathrm{~min}$ in traditional Fenton process, COD, chroma and $\mathrm{UV}_{254}$ removal reached $23.6 \%$, $48.5 \%$ and $25.6 \%$, respectively. With the reaction time increased to $90 \mathrm{~min}$, COD, chroma and $\mathrm{UV}_{254}$ removal reached $48.7 \%$, 95.2\% and 50.3\%, respectively. The oxidation mechanism by Fenton's reagent was due to highly reactive hydroxyl radical generated in an acidic solution by the catalytic decomposition of $\mathrm{H}_{2} \mathrm{O}_{2}$. The results indicated that increasing the reaction time would produce more hydroxyl radicals, which could enhance the oxidation degradation of organic compounds in leachate. In microwave enhanced Fenton process, COD, chroma and UV $\mathrm{VV}_{254}$ removal reached $43.9 \%$, 93.8\% and $47.4 \%$ within just 10 minutes, which was far above the removal in traditional Fenton process within the same reaction time. Microwave irradiation of 10 minutes brought a close removal with 90 min's Fenton's process. The accelerated and enhanced organic pollutants removal by microwave irradiation should be ascribed to the fast and selective heating of water and $\mathrm{H}_{2} \mathrm{O}_{2}$ molecules. Higher temperatures will accelerate the generation rate of hydroxyl radicals and will also increase the reaction possibility between hydroxyl radicals and organic pollutants [5].

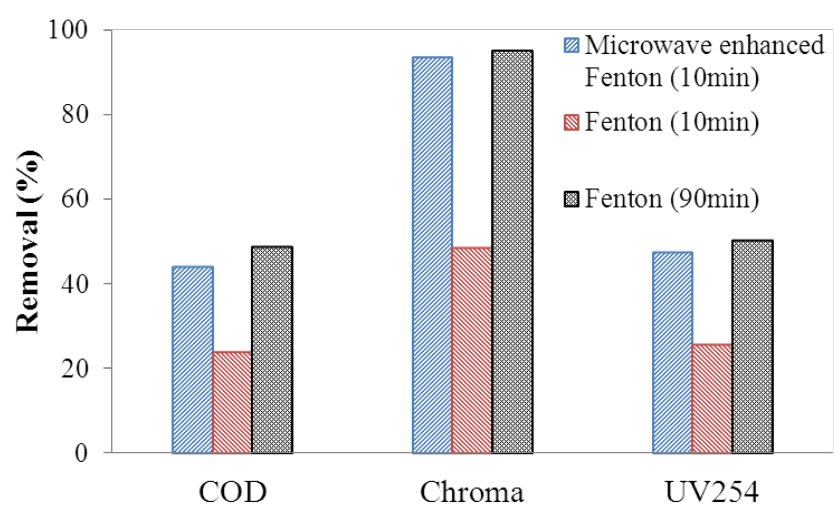

Fig. 1 Removal of COD, Chroma and $\mathrm{UV}_{254}$ in leachate by Fenton process and microwave-enhanced Fenton process 


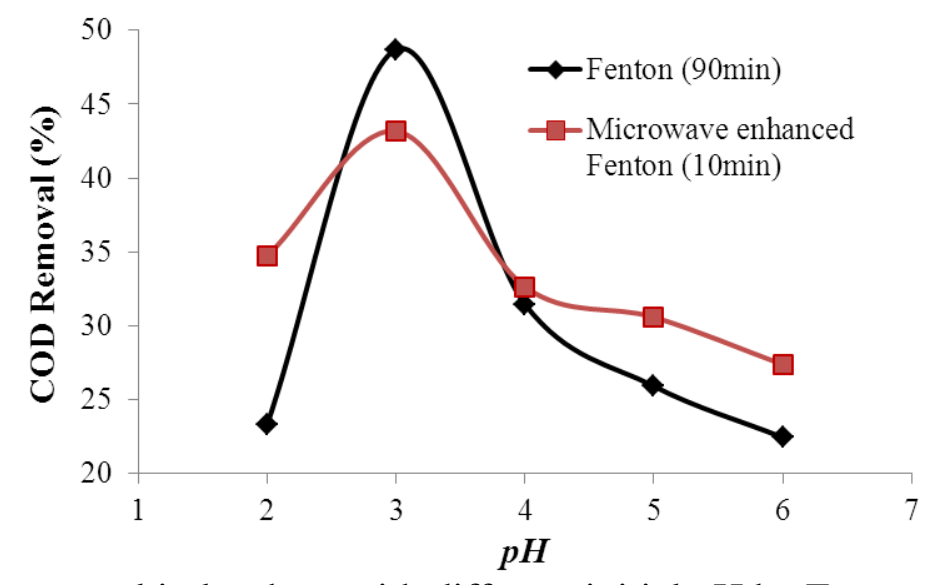

Fig. 2 COD removal in leachate with different initial $\mathrm{pH}$ by Fenton process and microwave-enhanced Fenton process

Influence of $\mathrm{pH}$ on COD removal in Fenton process and microwave-enhanced Fenton process is shown in Fig. 2. The initial $\mathrm{pH}$ value is very important for the efficiency of Fenton process, and maximum COD removal efficiencies occurred at $\mathrm{pH}$ 3.0. At low $\mathrm{pH}(<3.0)$, COD removal efficiency decreased sharply, due principally to the lower reaction rate of $\mathrm{Fe}^{2+}$ and $\mathrm{H}_{2} \mathrm{O}_{2}$, the increased scavenging of $\bullet \mathrm{OH}$ by $\mathrm{H}^{+}$, and the increased inhibit on the reaction between $\mathrm{Fe}^{3+}$ and $\mathrm{H}_{2} \mathrm{O}_{2}$ due to $\mathrm{H}^{+}$. On the other hand, COD removal dropped significantly with increasing $\mathrm{pH}(>3.0)$, due to the increasing rate of auto decomposition of $\mathrm{H}_{2} \mathrm{O}_{2}$, deactivation of a ferrous catalyst with the formation of ferric hydroxo complexes, and the decreased oxidation potential of $\bullet \mathrm{OH}$. Influence of $\mathrm{pH}$ on COD removal was less significant in microwave-enhanced Fenton process than in Fenton process, which should be ascribed to accelerating the generation rate of hydroxyl radicals and reaction reactivity between hydroxyl radicals and organic pollutants by microwave irradiation [8].

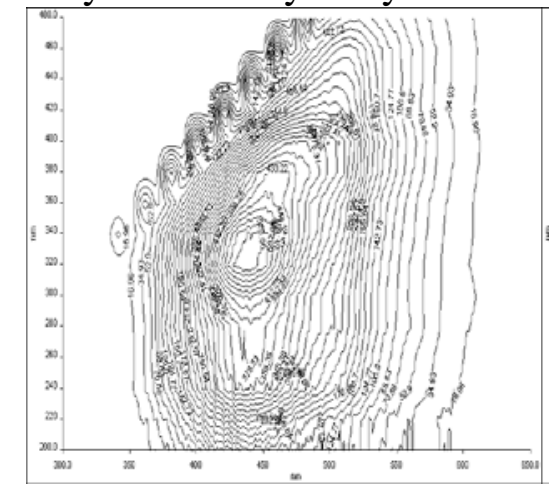

(a)

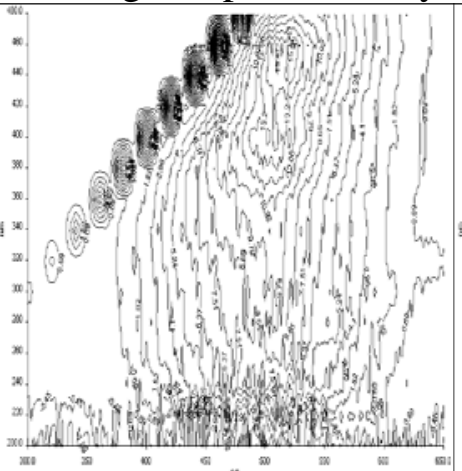

(b)

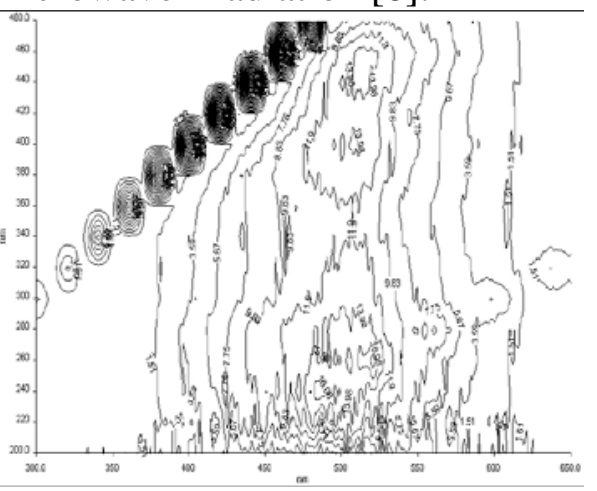

(c)

Fig. 3 The EEMs of leachate (a) and leachate treated by Fenton process(90min) (b) and microwave-enhanced Fenton process (10min) (c)

EEMs can provide spectral information about the organic matter compositions of leachate sample before and after treatment (Fig. 3). There were several typical fluorescence peaks in leachate [9][10]: Fulvic-like (Ex/Em=380 390/450 470nm, 390 410/480 500nm), Visible fulvic-like (Ex/Em=300 350/450 470nm, 290 310/410 420nm), UV fulvic-like (Ex/Em=215 225/450 460 nm, 240 260/430 450nm). Those fluorescence peaks were almost completely disappeared after Fenton process or microwave-enhanced Fenton process, which indicated that large molecular substances with strong fluorescence were decomposed into small substances without fluorescence or with weak fluorescence. The fluorescence intensity level after microwave-enhanced Fenton process was very low and well below Fenton process, which confirmed that microwave irradiation could enhance the degradation of organic compounds in leachate. 


\section{Conclusion}

Microwave irradiation can promote the generation rate of hydroxyl radicals and reaction reactivity between hydroxyl radicals and organic pollutants, so it can remarkably accelerate and enhance the decomposition of organic pollutants in landfill leachate. The removal of COD, $\mathrm{UV}_{254}$, chroma and EEMs peaks are superior in microwave-enhanced Fenton process, and the process has much higher decomposition efficiency and less $\mathrm{pH}$ influence than single Fenton process.

\section{Acknowledgement}

In this paper, the research was financially supported by the National Natural Science Foundation of China (No. 51308284).

\section{References}

[1] Bradley O. Clarke, Tarun Anumol, Morton Barlaz, Shane A. Snyder. Investigating landfill leachate as a source of trace organic pollutants [J]. Chemosphere, 2015 127c 269-275.

[2] Qi-Qi Zhang, Bao-Hu Tian, Xuan Zhang, Abbas Ghulam, Cheng-Ran. Investigation on characteristics of leachate and concentrated leachate in three landfill leachate treatment plants [J]. Waste Management, 201333 (11) 2277-2286.

[3] S. Renou, J.G. Givaudan, S. Poulain, F. Dirassouyan, P. Moulin. Landfill leachate treatment: Review and opportunity [J]. Journal of Hazardous Materials, 2008150 (3) 468-493.

[4] Yang Deng. Physical and oxidative removal of organics during Fenton treatment of mature municipal landfill leachate [J]. Journal of Hazardous Materials, 2007146 (1-2) 334-340.

[5] Shu-Ting Liu, Jiao Huang, Ying Ye, Ao-Bo Zhang, Li Pan, Xue-Gang Chen. Microwave enhanced Fenton process for the removal of methylene blue from aqueous solution [J]. Chemical Engineering Journal, 2013215 (2) 586-590.

[6] Yu Yang, PengWang, Shujie Shi, Yuan Liu. Microwave enhanced Fenton-like process for the treatment of high concentration pharmaceutical wastewater [J]. Journal of Hazardous Materials, 2009168 (1) 238-245.

[7] APHA, AWWA, WEF, 1998. Standard Methods for the Examination of Water and Wastewater, 20th ed. American Public Health Association, Washington, DC.

[8] Yun whan Kang and Kyung-yub Hwang. Effects of reaction conditions on the oxidation efficiency in the fenton process. Water Research, 2000 34(10) 2786-2790.

[9] ZhiPing Liu, WenHui Wu, Ping Shi, JinSong Guo, Jin Cheng. Characterization of dissolved organic matter in landfill leachate during the combined treatment process of air stripping, Fenton, SBR and coagulation [J]. Waste Management, 201541 (1) 111-118.

[10] Sarah E.H. Comstock, Treavor H. Boyer, Katherine C. Graf, Timothy G. Townsend. Effect of landfill characteristics on leachate organic matter properties and coagulation treatability. Chemosphere, 201081 (7) 976-983. 